\title{
Measurement of temperature and velocity fluctuations in a turbulent diffusion flame by optical fiber thermometer and laser 2-focus velocimeter
}

\author{
Hironobu Ueki ${ }^{\text {a }}$, Masahiro Ishida ${ }^{a}$, and Daisaku Sakaguchi ${ }^{\text {a }}$ \\ ${ }^{a}$ Department of Mechanical Systems Engineering, Faculty of Engineering, \\ Nagasaki University, 1-14 Bunkyo-machi Nagasaki 852 Japan
}

\begin{abstract}
Both fluctuations of local velocity and local temperature were measured in a steady turbulent diffusion flame of propane by using the semiconductor laser 2-focus velocimeter (L2F) and the optical fiber thermometer (OFT) respectively. The flame temperature and the soot particle density were calculated by applying the infrared two-color method to the measured radiant energy from the soot particles in the flame. In the analysis of the frequency power spectra of temperature and velocity fluctuations, the correlation-based slotting technique was adopted for those data with the nonuniform time interval. It is shown that the time mean value and the fluctuation of the flame temperature decrease gradually toward downstream in the luminous flame region, and those of the soot density increase due to decay of turbulence along the flame axis. On the other hand, both time mean and fluctuation of the flame temperature increase in the radial direction from the center to the periphery due to the effect of air entrainment marked in the peripheral region of the flame. Furthermore, the power spectrum of the velocity fluctuation is not always the same as that of the temperature fluctuation in the flame center.
\end{abstract}

Key words: Diffusion flame, Temperature, Soot, Velocity, Fluctuation, Spectrum

\section{INTRODUCTION}

In order to clarify the structure of turbulent diffusion flame, image measurement ${ }^{1}$, temperature measurements by laser Rayleigh scattering ${ }^{2}$, and by thermocouple with thin wire ${ }^{3}$ have been reported. The authors ${ }^{4}$ have revealed the temporal variation and the spacial distribution of the flame temperature and the soot density in the combustion chamber of a direct injection diesel engine under various operating conditions by means of the optical fiber thermometer (OFT) which consists of the optical sensor made of a sapphire rod. And, the authors also clarified the applicability ${ }^{5,6}$ of the semiconductor laser 2 -focus velocimeter (L2F) to measurement of the turbulent velocity field in a centrifugal blower.

In the present study, in order to understand more easily the processes of soot formation and soot oxidization in the diesel combustion, the basic structure or turbulence characteristics of a steady turbulent diffusion flame of propane was examined experimentally by measuring the gas velocity fluctuation and the flame temperature fluctuation under the luminous flame

\section{Further author information -}

H. U. (correspondence): E-mail: ueki@net.nagasaki-u.ac.jp; WWW: URL: http://tfl.mech.nagasaki-u.ac.jp/;Telephone: +81-958-47-1111, ext.2649; Fax: +81-958-47-3247

M. I. : E-mail: hiro@net.nagasaki-u.ac.jp

D. S. : E-mail: dai@tfl.mech.nagasaki-u.ac.jp 
condition which is usually seen in diesel engine combustion chamber. Measurements were conducted by using the above two non-intrusive measuring instruments, that is, the L2F for velocity measurement and the OFT for temperature measurement. The difficulty in these measurements is to be not easy for obtaining the continuous data with uniform time interval because the flame is not always luminous especially in the peripheral region of the flame even in the case of diffusion flame and the seeding particle for velocity measurement is supplied randomly into the measuring volume. In order to analyze accurately the frequency spectra of temperature and velocity fluctuations from the data with nonuniform time interval, the correlation-based slotting (CBS) technique ${ }^{7,8}$ was adopted for both temperature and velocity data.

\section{MEASUREMENT AND ANALYSIS}

A steady turbulent diffusion flame was formed by using the burner with a circular nozzle of $3 \mathrm{~mm}$ in diameter. A small amount of air was inducted in the propane gas for supplying the seeding particles which is inevitable for velocity measurement by the L2F. Therefore, the flame in the present experiment is not a complete diffusion one but a premixed one with a rich propane concentration. The equivalence ratio of the propane mixture was 17.5 and the Reynolds number was 8,830 . Figure 1 (a) shows the system diagram of the flame temperature measurement consisting of the optical fiber thermometer Model 100C manufactured by Accufiber Corp. The radiant energy from the luminous flame was concentrated on the tip of the optical fiber with a diameter of $0.6 \mathrm{~mm}$ through two lenses, and the radiant energy of the two wave lengths of 800 and $950 \mathrm{~nm}$ was selected and converted to each electric voltage output. These voltage outputs were sampled by the transient combustion analyzer CB-467 manufactured by Ono Sokki Ltd., and were transferred to workstations (HP715/33 and HP715/75) for the calculation of the flame temperature and the KL value based on the two-color method ${ }^{9}$. The KL value is the index which represents the density of the soot particles in the measuring volume. The axes in the measuring volume are as follows; the $z$-axis is the direction of fuel gas flow, the $y$-axis corresponds to the optical fiber sensor axis, and the $x$-axis is taken perpendicular to both the $z$-and $y$ -axes. The radiant energy of the focal point is led to the optical fiber correctly, however, the radiant energy from outside of the focal point might be included in a part. Figure 1(b) shows the size of the measuring volume estimated from the geometrical optics under the condition of the present lens system for temperature measurement. The shape of the measuring volume having the radiant energy level of $100 / \mathrm{e} \%$ of that of the focal point is a revolution of ellipse which has the major axis length of $6.6 \mathrm{~mm}$

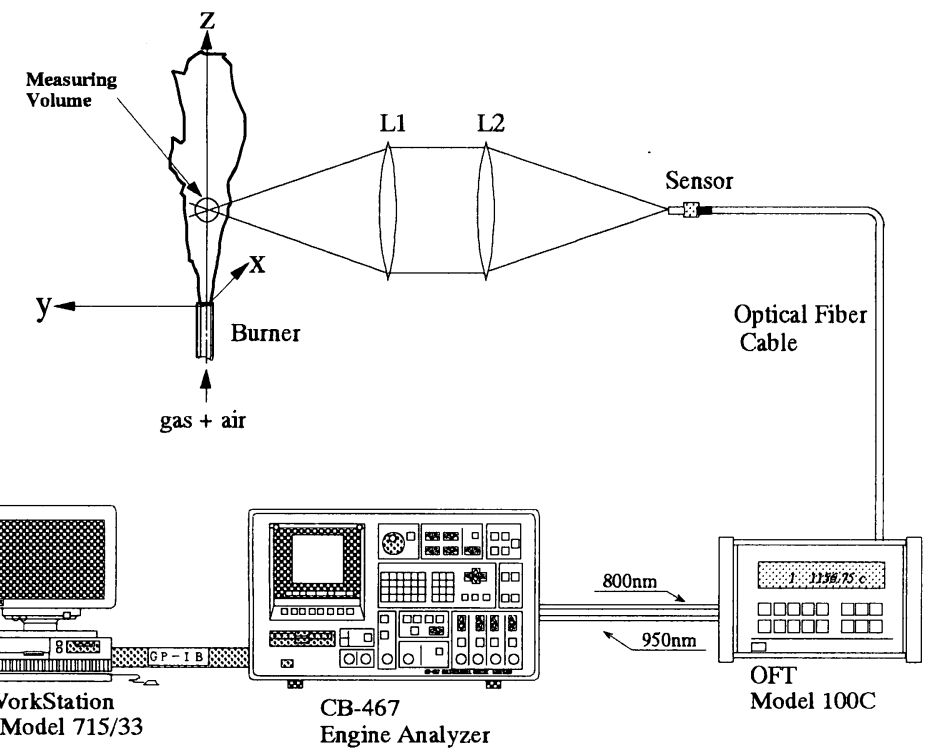

(a) System diagram of OFT

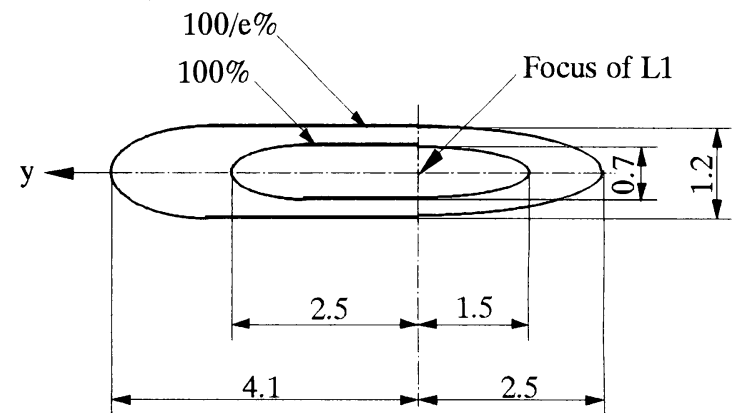

(b) Measuring volume

Fig. 1 Temperature measurement system 
in the $y$-direction and the minor axis diameter of $1.2 \mathrm{~mm}$ in the $x, z$-directions. The length between the focal point and the left edge of the measuring volume is larger than that between the focal point and the right edge.

Figure 2 (a) shows the system diagram of the laser 2-focus velocimeter (L2F) for the gas flow velocity measurement. A widestripe semiconductor laser with the maximum output power of $1 \mathrm{~W}$ was installed as the light source. This semiconductor laser was an infrared type with a wave length of $810 \mathrm{~nm}$. The diverging light from the semiconductor laser was led to a collimating lens and was divided into two beams with a very small difference in angle by the beam splitter. In order to form the measuring volume, the condenser lens with a focal length of $40 \mathrm{~mm}$ transferred two beams to two foci. The back-scattered lights from a seeding particle at each focus were led to each Si-avalanche photodiode through a collimating lens and a microscope objective. The diameter of the sensible area of the Si-avalanche photodiode was $3.0 \mathrm{~mm}$. The scattered light was converted to an electric pulse by the Si-avalanche photodiode and was led to the time-of-flight counter through an amplifier. The timer counted the time when a time-of-flight datum acquired. The time-of-flight data and the timer data were acquired simultaneously by a personal computer through a digital interface with the maximum sampling rate of $100 \mathrm{kHz}$. The optical system was $350 \mathrm{~mm}$ long including the light source. Figure 2 (b) shows the measuring volume consisting of two sheet-shaped foci facing each other. The distance $S$ between two foci was about $0.09 \mathrm{~mm}$, the thickness $L$ of the focus was about $0.01 \mathrm{~mm}$ and the width $w$ was about 0.25 $\mathrm{mm}$ in the $x^{\prime}$-direction. Two foci are almost parallel each other. In order to form the observation area with the uniform light intensity in the center part of the focus region, the slit with the width of $0.2 \mathrm{~mm}$, the length of $3 \mathrm{~mm}$ and the thickness of 0.013 $\mathrm{mm}$ was mounted in front of the photodiodes. The corresponding observation area at the focus was about $0.01 \mathrm{~mm}$ thick, because the microscope objective of 20 magnifications was used for expanding the focus image. Silicon dioxide powder with a mean diameter of $2 \mu \mathrm{m}$ was used as the seeding particle for the flow measurement by the L2F.

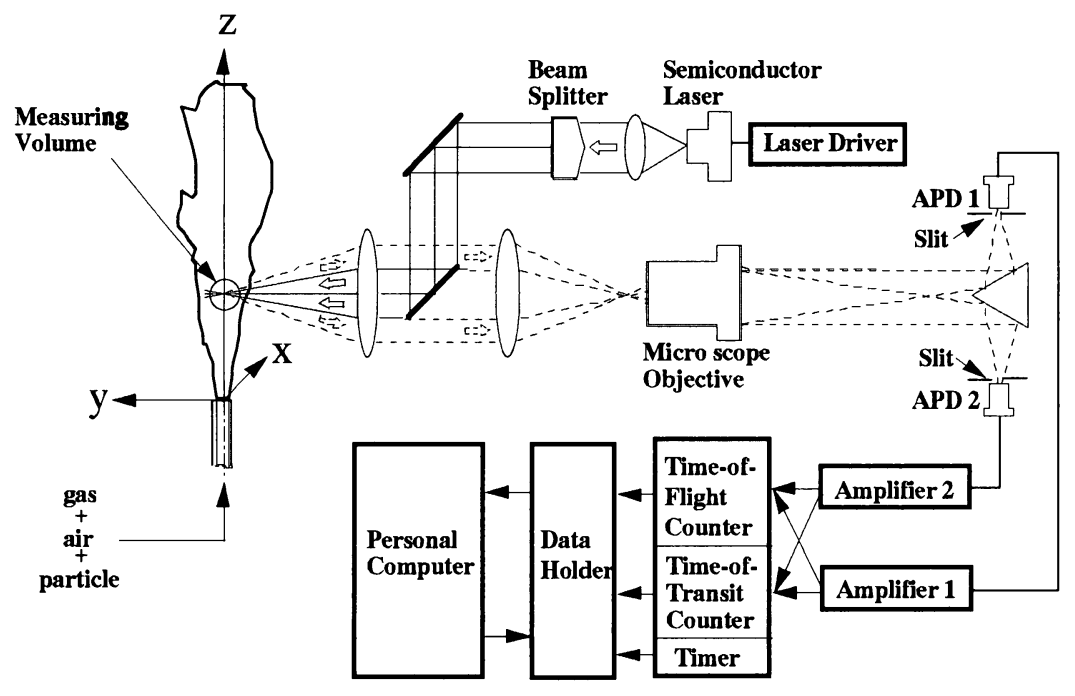

(a) System diagram of L2F

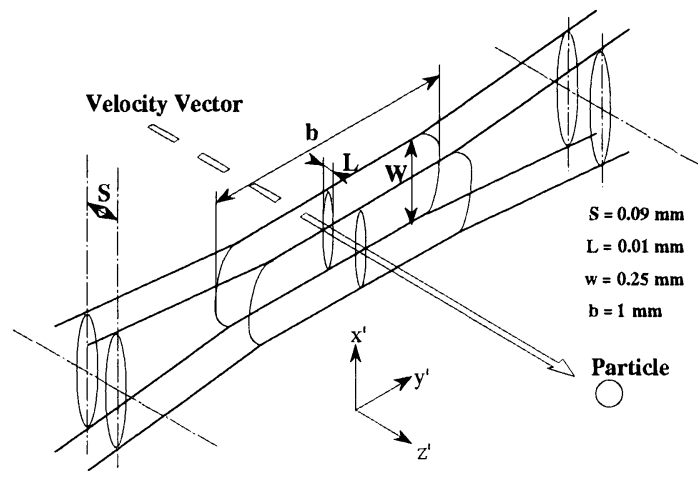

(b) Measuring volume

Fig. 2 Flow velocity measurement system

\section{TIME HISTORY OF TEMPERATURE}

The maximum height of the tested diffusion flame was about $450 \mathrm{~mm}$, and the maximum diameter of the flame was about 50 $\mathrm{mm}$. In the region of $z>90 \mathrm{~mm}$, the luminous flame was observed. Figure 3 shows an example of the temporal variation of the 
OFT output voltage, and the calculated flame temperature and the $\mathrm{KL}$ value at the measuring point $(x, y, z)=(0,-10,300)$. The total measuring time $\Delta T$ was $72 \mathrm{~ms}$, and the data sampling frequency was $20 \mathrm{kHz}$. The temperature fluctuates highly near the measuring time $T$ of around $64 \mathrm{~ms}$ due to a very low voltage output of the OFT. At this time, no luminous flame must be in the measuring volume judging from both the OFT output and the KL value. The reliability of the measured temperature calculated from the extremely low OFT output is not enough for the temperature fluctuation analysis. Therefore, the flame temperature can be conditionally sampled only when the luminous flame must be in the measuring volume. This is a similar situation of the

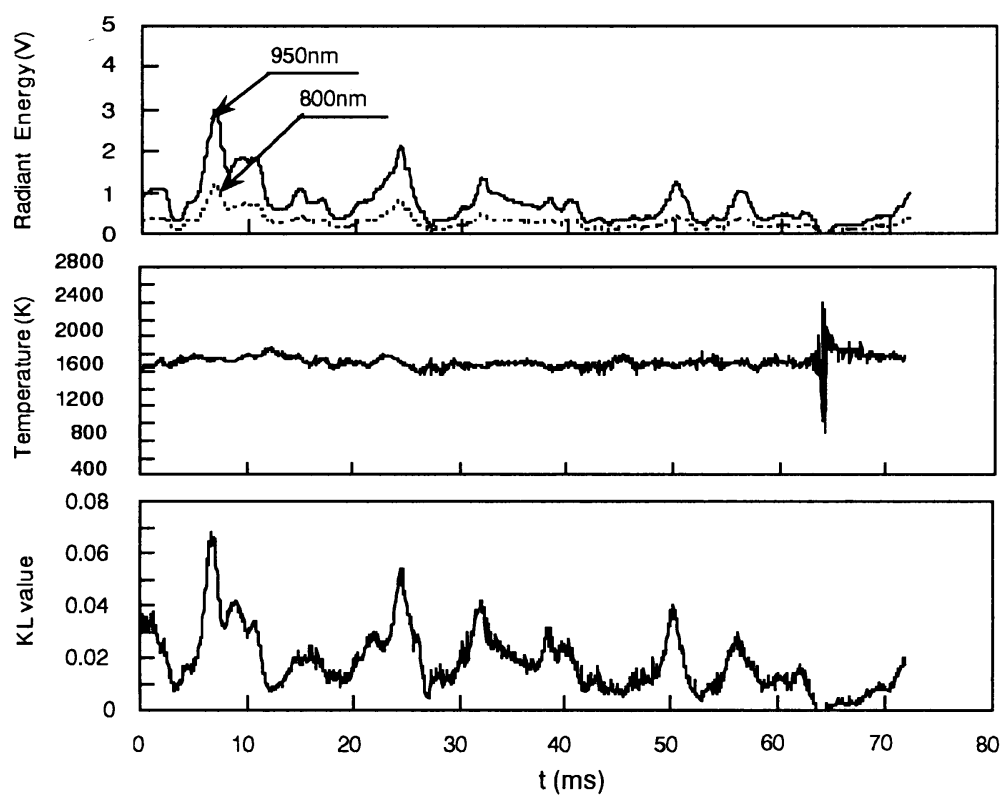

Fig. 3 Time history of OFT output, temperature and KL value

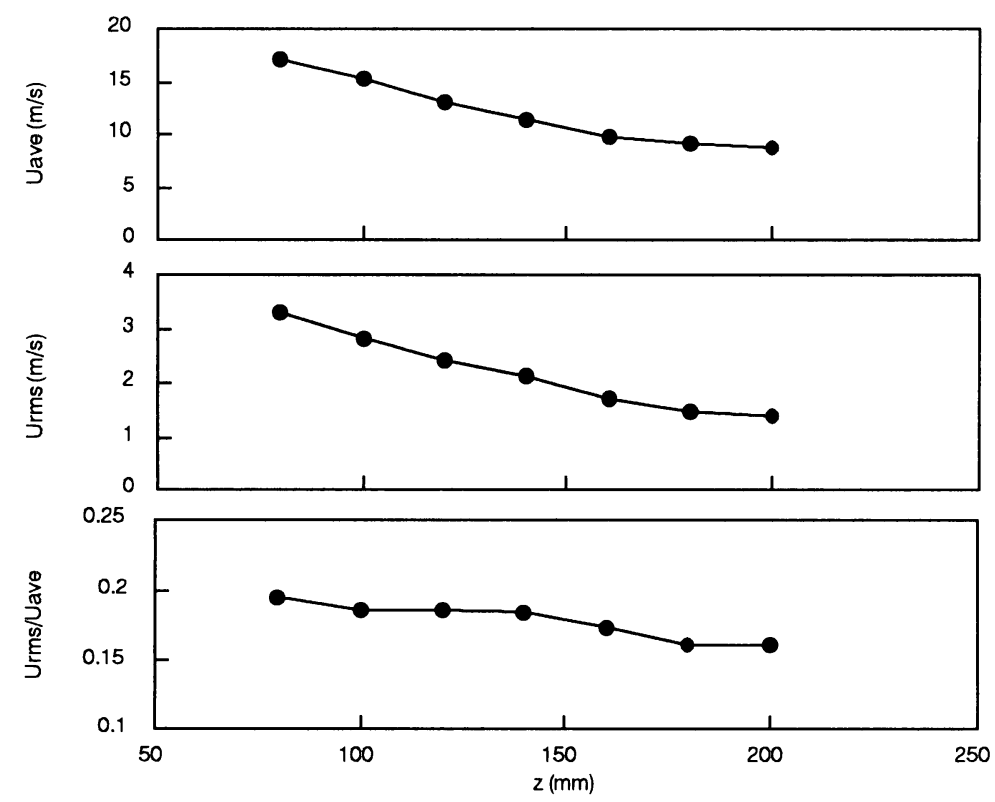

Fig. 4 Variation of velocity along the flame axis

(Mean velocity, velocity fluctuation, and intensity of velocity fluctuation) 
velocity measurement by the $\mathrm{L} 2 \mathrm{~F}$ because the laser velocimeters can obtain the data only when the seeding particle comes into the measuring volume.

\section{VELOCITY AND TEMPERATURE DISTRIBUTIONS ALONG THE FLAME AXIS}

Figure 4 shows the measured spatial distributions of time mean velocity $U_{a v e}$, fluctuation velocity $U_{r m s}$, and fluctuation intensity $U_{r m s} / U_{\text {ave }}$ along the flame axis. The number of effective data sampled was 32,000 at each measuring point. Mean velocity and velocity fluctuation decreased gradually toward downstream along the flame axis. It is expected that the momentum of the fuel jet was transferred to the entrained air, and that the effect of gas expansion by combustion was not remarkable in the measured region. Flow velocity could not measured in the region higher than $200 \mathrm{~mm}$ in the $z$-direction because of the short focus length of $43 \mathrm{~mm}$ in the focusing lens of the present L2F system.

Figure 5 shows the spatial distributions of time mean temperature $T_{\text {ave }}$, temperature fluctuation $T_{r m s}$, and fluctuation intensity $T_{r m s} / T_{\text {ave }}$ of the measured temperature along the flame axis. The number of effective data sampled was 14,400 at each measuring point. In the measured region between 125 and $300 \mathrm{~mm}$ in the flame height, the time mean temperature and the temperature fluctuation decreased gradually along the flame axis partly due to the entrainment of ambient air and partly due to the decay of turbulence based on a decrease in flow velocity. Furthermore, the flame temperature in the region lower than $125 \mathrm{~mm}$ could not measured because of too low OFT output, and the temperature in the region higher than $300 \mathrm{~mm}$ is not shown here because the measured temperature was highly disturbed by a large intermittent fluctuation.

Figure 6 shows the spatial distributions of time mean $K L$ value indicated by $K L_{a v e}$, fluctuation $K L_{r m s}$, and fluctuation intensity $K L_{m s} / K L_{\text {ave }} \mathrm{KL}$ along the flame axis. The luminous flame does not appears in the region lower than $125 \mathrm{~mm}$ judging from that the value of $K L_{\text {ave }}$ was almost zero. It is noticeable that the time mean $K L$ value and its fluctuation increase along the flame axis to the contrary of decrease in velocity and temperature. It might be caused by that the oxygen concentration decreased downstream in the central part of the flame due to the decrease in turbulence even though air is entrained in the peripheral region of the flame. The mean KL value should become zero at the flame top. In Fig.6, the $K L_{r m s}$ increased with the $K L_{\text {ave }}$, however, the

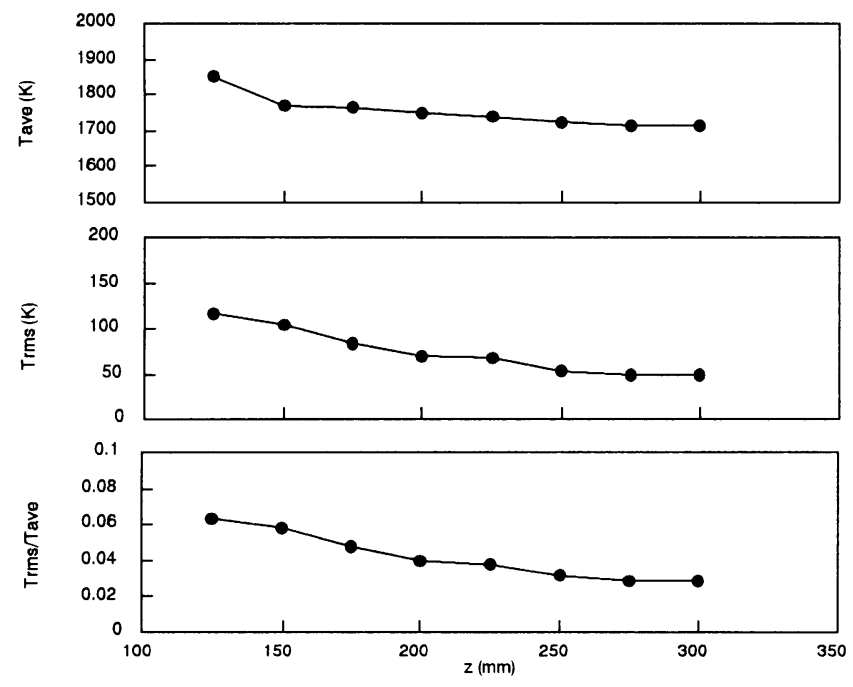

Fig. 5 Variation of temperature along the flame axis (Mean temperature, temperature fluctuation, and fluctuation intensity)
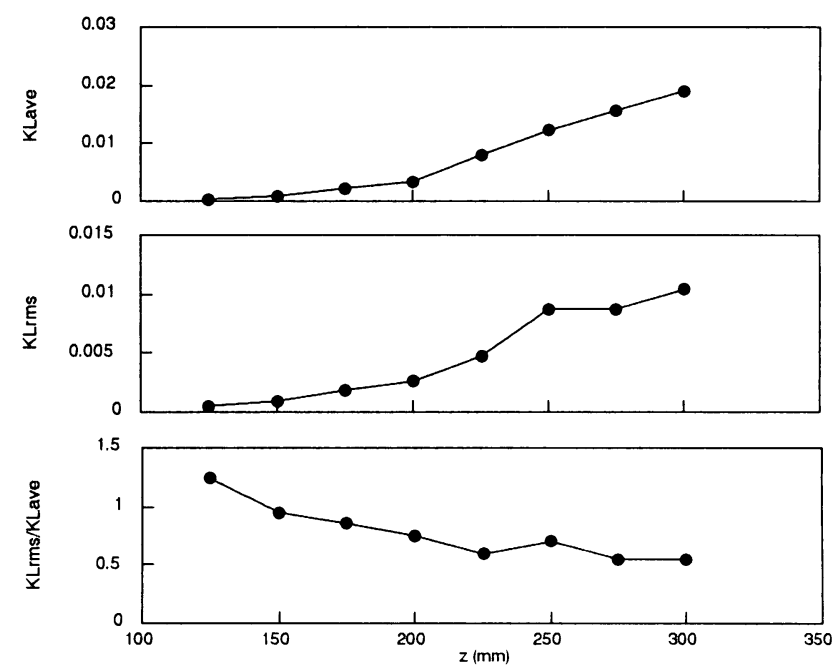

Fig. 6 Variation of KL value along the flame axis (Time mean KL, fluctuation of $\mathrm{KL}$, and fluctuation intensity of KL) 
fluctuation intensity $K L_{r m s} / K L_{\text {ave }}$ decreased gradually along the direction of gas flow. Furthermore, near the position where the luminous flame firstly appears, the fluctuation intensity $K L_{r m s} / K L_{a v e}$ is very high having the order of unity.

The correlation-based slotting (CBS) technique proposed by Roberts et al. ${ }^{7}$ can analyze the turbulence spectrum from the data with nonuniform time interval. This CBS technique was adopted for analyzing the turbulence spectrum of both the data of flow velocity and temperature. Figure 7 shows the power spectra of temperature measured at 150, 225 and $300 \mathrm{~mm}$ high on the flame axis. The data sampling rate was $20 \mathrm{kHz}$, however, the maximum analyzing frequency was set $2 \mathrm{kHz}$ in order to estimate the spectrum accurately. The power level in the range from $10^{2}$ to $10^{3} \mathrm{~Hz}$ decreased markedly in the spectra obtained at $\mathrm{z}=225$ and $300 \mathrm{~mm}$ compared with the spectrum at $\mathrm{z}=150 \mathrm{~mm}$. The straight line shown in Fig. 7 shows the $-5 / 3$ power law of the isotropic turbulence. Judging from that the inclination of the power spectrum is almost the $-5 / 3$ power law near the frequency of $10^{3} \mathrm{~Hz}$, the temperature fluctuation has a characteristics of the isotropic turbulence. The power spectrum of temperature

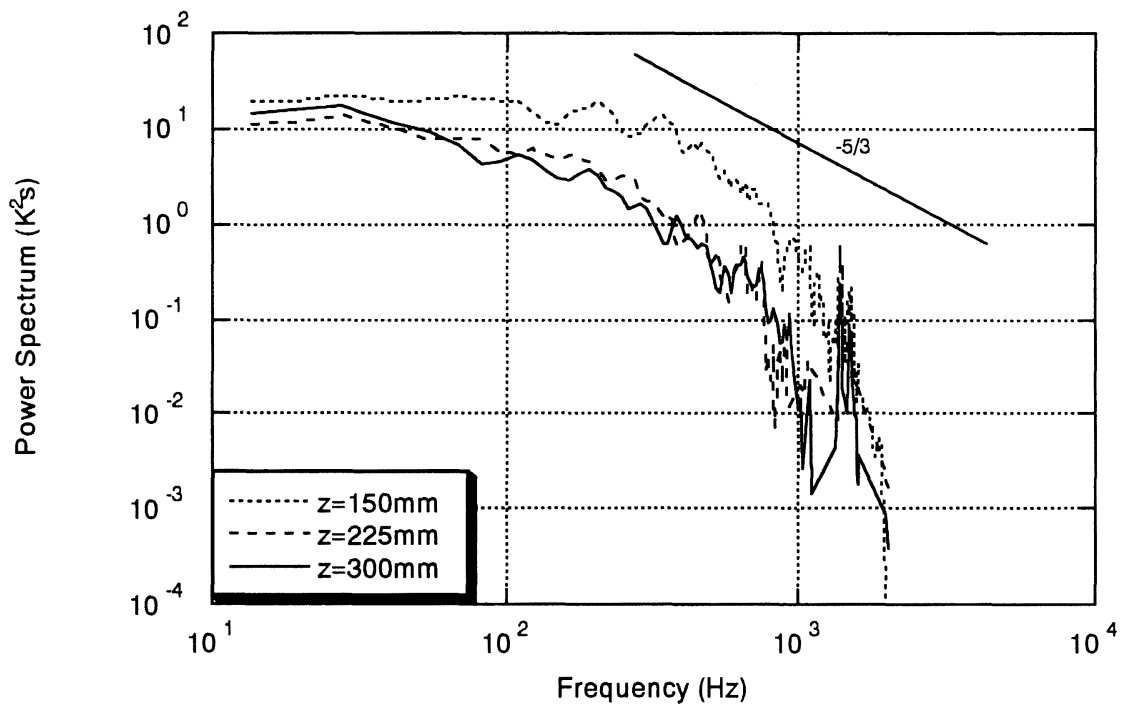

Fig. 7 Power spectrum of temperature along the flame axis

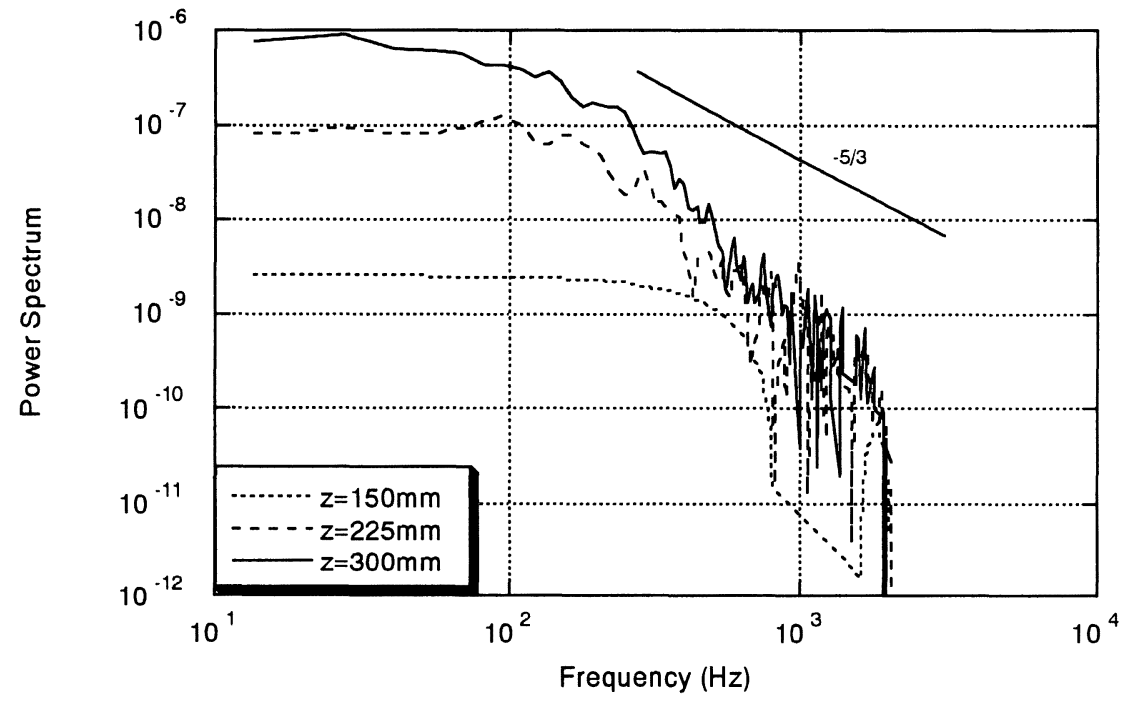

Fig. 8 Power spectrum of KL along the flame axis 


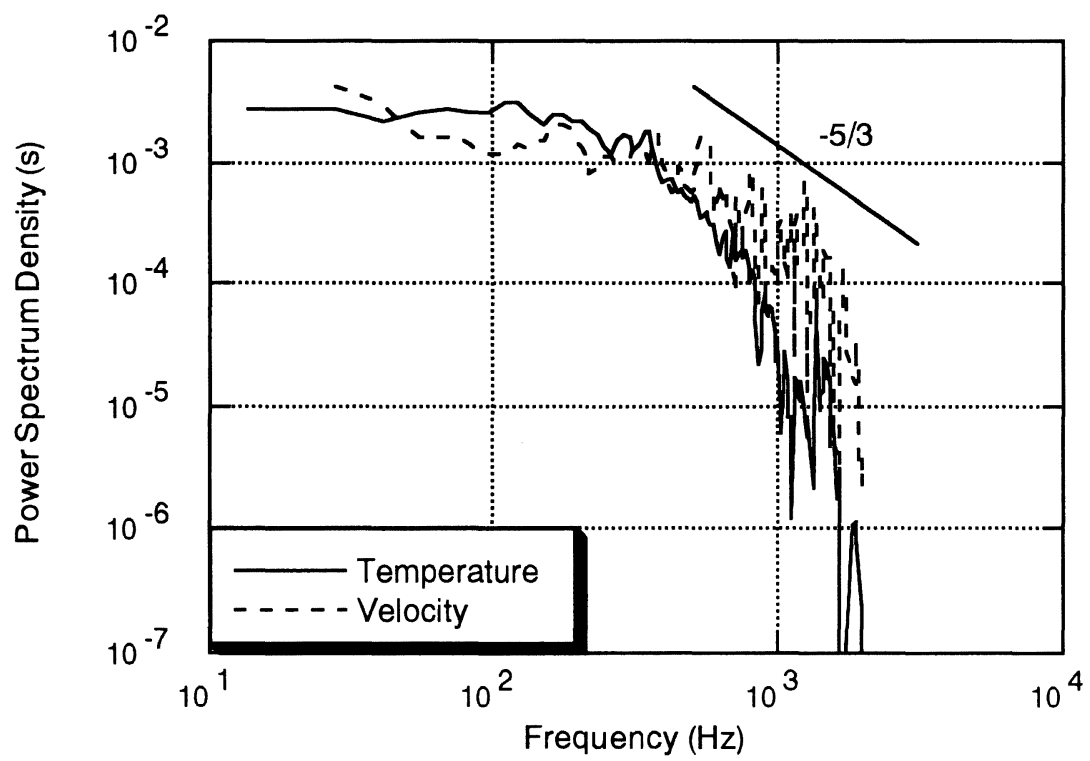

Fig. 9 Comparison of power spectrum density between velocity and temperature

fluctuation measured at $z=150 \mathrm{~mm}$ is almost flat in the frequency range smaller than $300 \mathrm{~Hz}$, and the power spectrum at $\mathrm{z}=225$ $\mathrm{mm}$ and $\mathrm{z}=300 \mathrm{~mm}$ decreased gradually from the frequency of $100 \mathrm{~Hz}$ which are similar as the result reported by Ida et al. ${ }^{10}$. Figure 8 shows the power spectra of the KL value at $z=150,225$, and $300 \mathrm{~mm}$. The power spectrum level of the KL increases remarkably in the frequency range smaller than $2 \mathrm{kHz}$ as the measuring position moves downstream because $K L_{r m s}$ increased along the flame axis. It is noticeable that the power spectrum at $\mathrm{z}=150 \mathrm{~mm}$ is almost flat in the frequency range smaller than $300 \mathrm{~Hz}$, on the other hand, the power spectra at $\mathrm{z}=225$ and $\mathrm{z}=300$ decreased gradually as the frequency increases. This behavior is similar as in the case of the temperature power spectrum shown in Fig.7.

Figure 9 shows a comparison of power spectrum density between velocity and flame temperature fluctuations measured at the point $(x, y, z)=(0,0,150)$. The number of velocity data used was 20,000 . The power spectrum density of the flame temperature is higher than that of the gas velocity at the frequency of $10^{2} \mathrm{~Hz}$, on the other hand, the power spectrum density of the gas

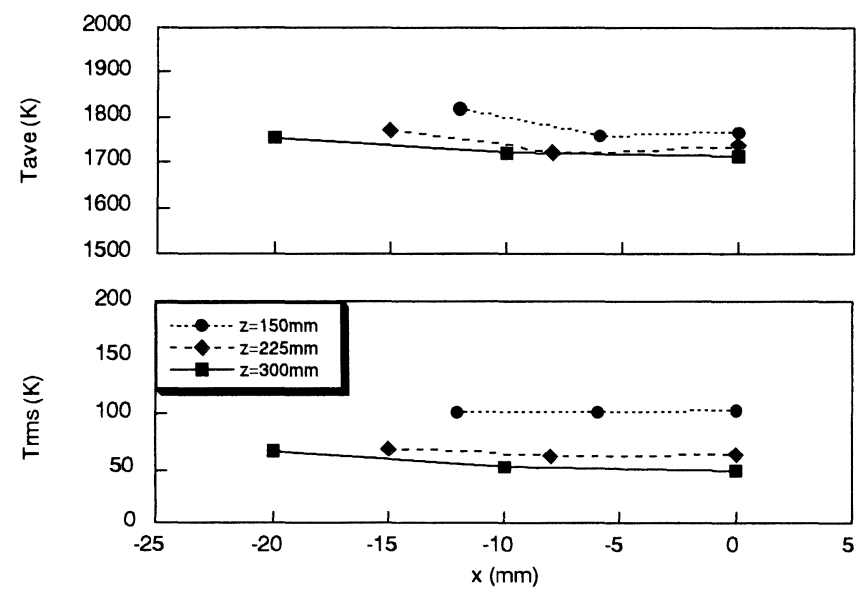

Fig. 10 Variation of temperature in the $\mathrm{x}$-direction
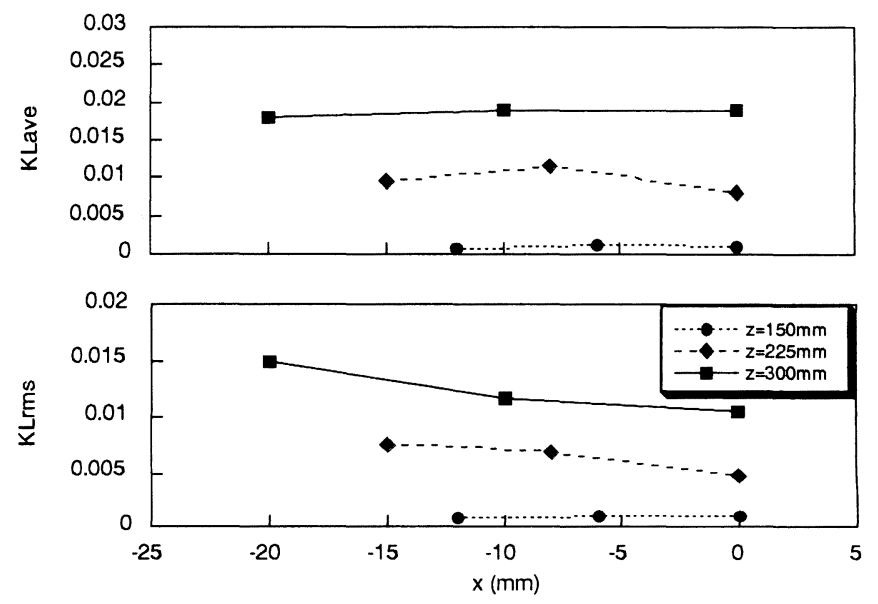

Fig. 11 Variation of $\mathrm{KL}$ in the $\mathrm{x}$-direction 
velocity is higher than that of the flame temperature near the frequency of $10^{3} \mathrm{~Hz}$. The correlation between the two fluctuations of velocity and temperature was not confirmed clearly. It seems to be necessary to acquire more number of data for the better spectrum analysis.

\section{TEMPERATURE VARIATION IN THE RADIAL DIRECTION}

Figure 10 shows the spatial distributions of time mean flame temperature $T_{\text {ave }}$ and fluctuation intensity of temperature $T_{r m s}$ in the $x$-direction at $z=150,225$, and $300 \mathrm{~mm}$. The left edge data in each $z$ position shown in Fig.10 indicates almost the periphery of the flame. In the measured region, both $T_{\text {ave }}$ and $T_{r m s}$ increased in the radial direction from the center to the periphery of the flame. These results shows that air entrainment is remarkable in the peripheral region of the flame.

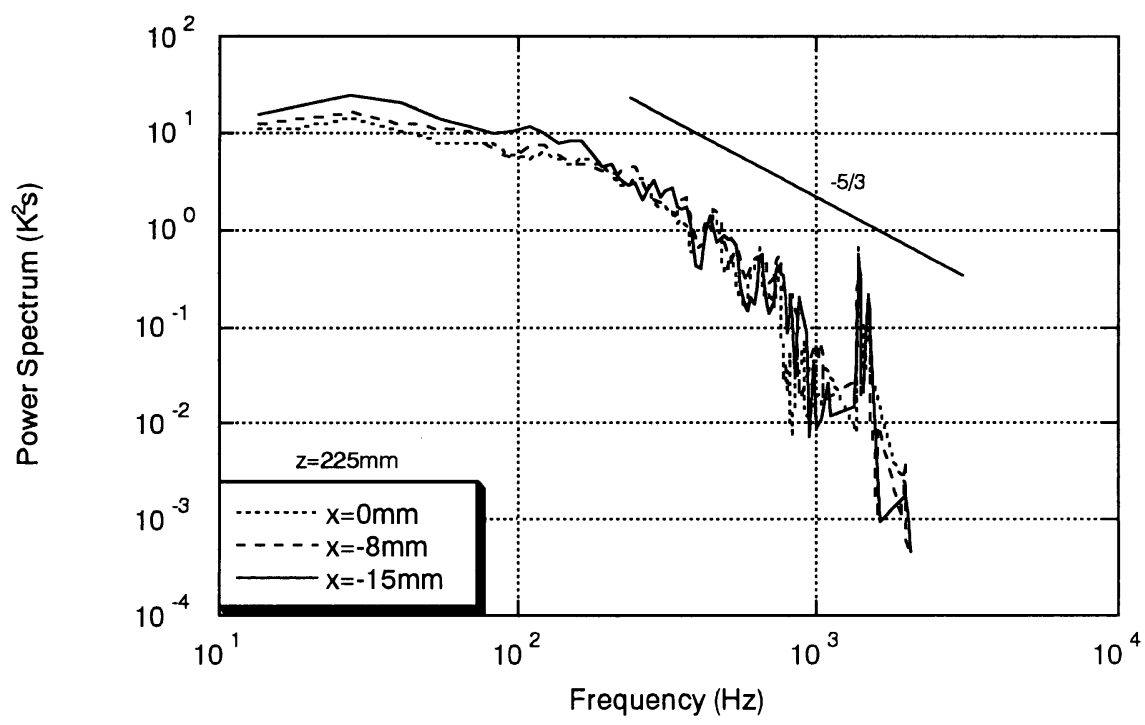

Fig. 12 Frequency power spectrum of temperature in the $\mathrm{x}$-direction

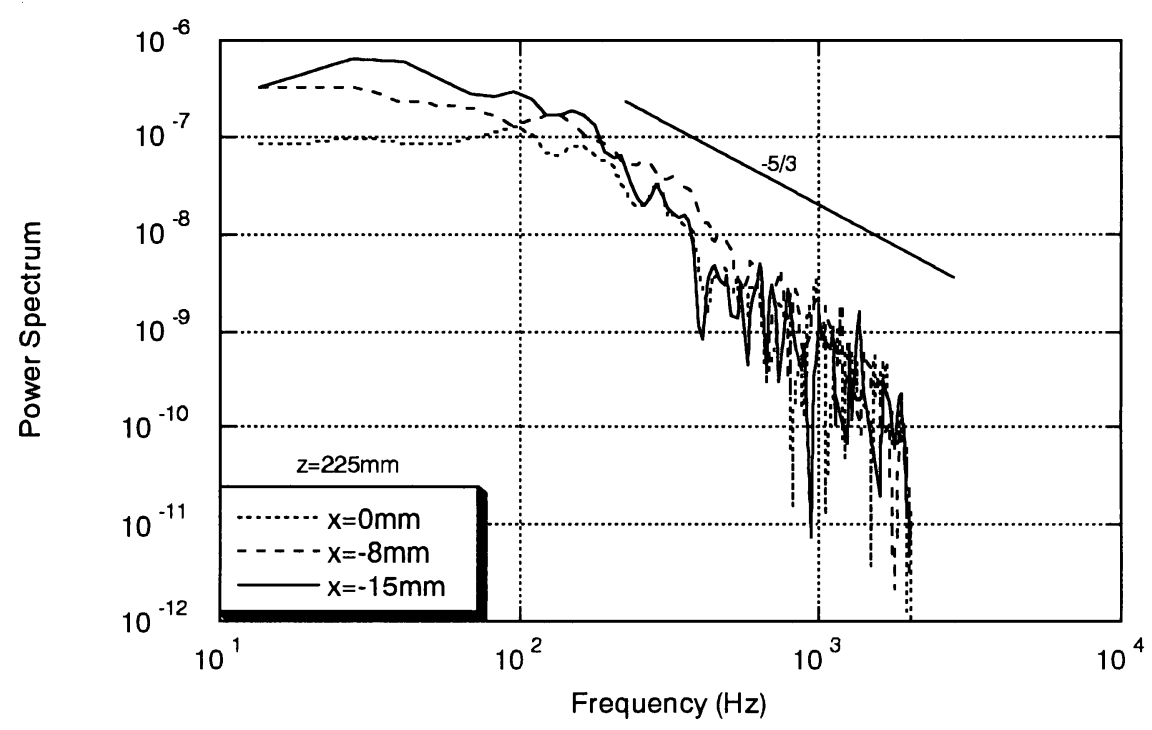

Fig. 13 Frequency power spectrum of $\mathrm{KL}$ in the $\mathrm{x}$-direction 

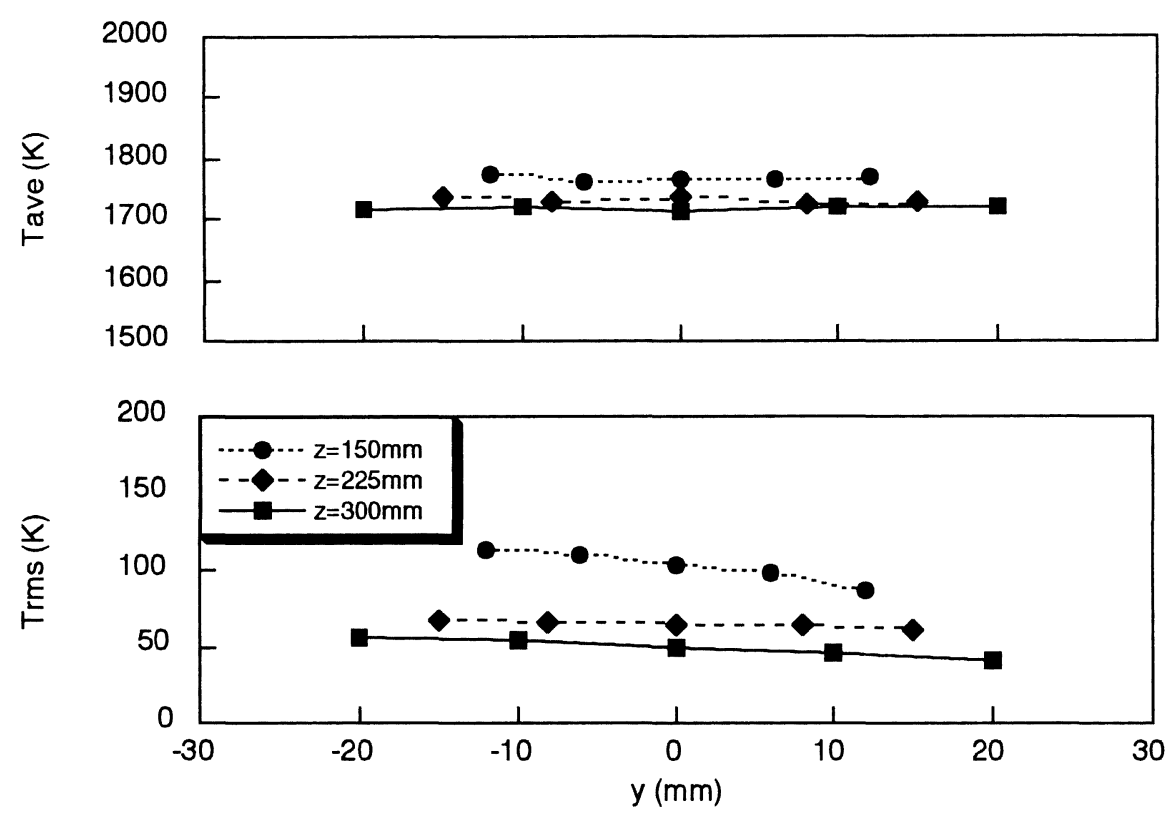

Fig. 14 Temperature distribution in the y-direction

Figure 11 shows the spatial distributions of time mean $K L$ value $K L_{\text {ave }}$ and $K L$ fluctuation $K L_{r m s}$ in the $x$-direction at $z=150$, 225 , and $300 \mathrm{~mm}$. The $K L_{\text {ave }}$ distribution is almost uniform in the flame section at each $z$ position, and the $K L_{r m s}$ increases in the radial direction which might be due to air entrainment.

Figure 12 shows comparison among the power spectra of the temperature fluctuation obtained at three radial positions in the section of $z=225 \mathrm{~mm}$. The power spectrum in the frequency range smaller than $200 \mathrm{~Hz}$ at $x=-15 \mathrm{~mm}$ is larger than that at $\mathrm{x}=0$ and -8. It might be caused by a remarkable temperature fluctuation due to the air entrainment vortex in the peripheral region of the flame. Figure 13 shows comparison among the power spectra of the KL fluctuation at the same positions shown in Fig.12. The KL power spectra are similar tendency as the flame temperature power spectra, that is, the power spectrum in the peripheral region of the flame is higher in the low frequency range.

Figure 14 shows the spatial distributions of the time mean flame temperature $T_{a v e}$ and the temperature fluctuation $T_{r m s}$ in the $y$ -direction at $z=150,225$, and $300 \mathrm{~mm}$. The radial variation of $T_{\text {ave }}$ in the $y$-direction is different from that of $T_{\text {ave }}$ in the $x$ direction shown in Fig.9, and the radial variation of temperature is not clear in the $y$-direction. On the other hand, the radial variation of the $T_{r m s}$ in the $y$-direction is similar as in the $x$-direction. This difference between the $x$-direction and the $y$ direction might be caused by that the measuring volume of the OFT is very small in the $x-z$ plane, on the other hand, it spreads largely in the $y$-direction. In other word, more than half of the measuring volume locates outside of the flame if the focal point is set at the periphery of the flame.

\section{CONCLUSION}

The temperature and the soot particle density in the luminous flame region of a turbulent diffusion flame was measured by the optical fiber thermometer based on the two-color method. The gas velocity in the flame was also measured accurately in the turbulent diffusion flame by means of the semiconductor laser 2-focus velocimeter developed by the authors. The correlationbased slotting technique was effectively adopted for the frequency power spectrum analysis of fluctuations of the flame 
temperature, the soot particle density and the gas velocity, which were the data with nonuniform time interval sampled only under the luminous flame condition.

The spatial distributions of the flame temperature and the soot particle density with respect to both time mean and fluctuation intensity could be measured accurately by the present optical system even under the condition that the measuring volume is several $\mathrm{mm}$ long along the optical axis. It is shown that the time mean value and the fluctuation of the flame temperature decrease gradually toward downstream in the luminous flame region, and those of the soot density increase due to decay of turbulence along the flame axis. On the other hand, both time mean and fluctuation of the flame temperature increase in the radial direction from the center to the flame periphery due to the effect of air entrainment marked in the peripheral region of the flame. Furthermore, the power spectrum of the velocity fluctuation is not always the same as that of the temperature fluctuation in the flame center.

\section{ACKNOWLEDGMENT}

The authors wish to thank to Mr. E. Hu and Mr. R. Toyohuku of Graduate School of Nagasaki University for their contribution on the experiment and the data analysis.

\section{REFERENCES}

1. T. Ida, K. Ohtake, "Time-Dependent Lagrangian Mixing Process in Turbulent Diffusion Flames observed using UltrahighSpeed Video System”, JSME Trans. (B), vol.61, No.589, pp.3329-3337, 1995 (in Japanese)

2. M. Komiyama, M. Matsui, T. Takagi, "Measurements of Instantaneous Radial Temperature Profiles in Turbulent Diffusion Flames”, Trans. JSME, Vol.60, No. 572(B), pp.1443-1448, 1994 (in Japanese)

3. H. Tanaka, A. Saima, "Experimental Study on the Flow Characteristics in Turbulent Diffusion Flames (2nd Report, Flow Characteristics in Turbulent Diffusion Flames, and the Effect of Charged Soot on Fluctuation Velocity)", Trans. JSME, Vol.58, No. 546(B), pp.386-392, 1992 (in Japanese)

4. M. Ishida, N. Matsumura, H. Ueki, G. Itoh, S. Kubota, J-G. Ko, "Measurement of Diesel Combustion by Optical Fiber Thermometer (1st. Report, Combustion Process and Exhaust Emission Level)", Trans. JSME, Vol.58, No. 555(B), pp.64823488, 1992 (in Japanese)

5. H. Ueki, M. Ishida, “Application of optoelectronic devices to a laser-2-focus velocimeter”, FED-vol.108, Fluid Measurement and Instrumentation Forum, ASME, pp.79-82, 1991

6. M.Ishida, H.Ueki, D.Sakaguchi, T.Surana, "Unstable Flow Measurement in a Centrifugal Blower by Semiconductor Laser 2Focus Velocimeter", Proc. of the 4th Asian International Conference on Fluid Machinery, Vol.1, pp.77-82, 1993

7. J. B. Roberts, D.B. S. Ajmani, "Spectral Analysis of Randomly Sampled Signals Using a Correlation-Based Slotting Technique”, IEEE Proc. Vol. 133, Pt.F, No.2, pp.153-162, 1986

8. H. Ueki and M.Ishida, "Turbulence Measurements in a Direct Injection Diesel Engine Combustion Chamber by LDV", SAE Paper No.891913, 1989

9. Y. Matsui, T. Kamimoto, S. Matsuoka, "A Study on the Application of the Two-Color Method to a Measurement of Flame Temperature and Soot Concentration in Diesel Engine”, SAE Paper No.800970, 1980

10. T. Ida, K. Ohtake, "Experimental Study of Turbulent Diffusion Flame Structure and Its Similarity", JSME International Journal, Series B, Vol.40, No.2, pp.304-311, 1997 